Indonesian Journal of Medicine (2019), 4(1): 54-58

https://doi.org/10.26911/theijmed.2019.04.01.09

\title{
Bilateral Simultaneous Rupture of the Quadriceps Tendon in a Healthy Elderly Woman: A Case Report
}

\author{
Pamudji Utomo'), Denny Adriansyah²) \\ 1)Department of Orthopaedic and Traumatology, Prof. Dr. R. Soeharso \\ Orthopaedics Hospital/Faculty of Medicine, Universitas Sebelas Maret \\ 2)Prof. DR. R. Soeharso Orthopaedic Hospital, Surakarta
}

\begin{abstract}
Background: Quadriceps tendon ruptures are uncommon injury that affects middle-aged women, predominantly it affects middle aged men. However, the bilateral quadriceps tendon rupture is extremely rare in the absence of systemic disease.

Subjects and Method: We reported a case of bilateral quadriceps tendon rupture in a healthy 65 year old female. She felt pain in both knees since 4 months before entering the hospital. Local physical examination on both knees reveal the loss of normal contour of rectus femoris, tenderness at superior pole patella the ruptur site, and palpable defect within $2 \mathrm{~cm}$ of superior pole patella. Patient unable to extend knee against resistance, and unable to perform straight leg raise. The patient was carried out a surgical repair with consent.

Results: After the surgery both knees were immobilized with knee extension splint for 6 weeks. Patient evaluation revealed no pain and progressive recovery of muscular strength after four months and could be able to walk and returned to normal activity.
\end{abstract}

Conclusion: Surgery is needed to repair quadriceps rupture.

Keywords: Quadriceps tendon, quadriceps rupture, repair tendon.

\section{Correspondence:}

Pamudji Utomo. Faculty of Medicine, Universitas Sebelas Maret. Jl. Ir. Sutami 36A, Surakarta, Central Java, Indonesia. Email: utomodr@yahoo.com.

\section{BACKGROUND}

$\overline{\text { Quadriceps tendon ruptures are uncommon }}$ injury that affect middle-aged women, predominantly it affects middle aged men (Clayton and Court-Brown, 2008). However, the bilateral quadriceps tendon rupture is extremely rare in the absence of systemic disease (Senevirathna et al., 2011; Ellanti et al., 2012). The rupture occurs from a powerful contraction of the quadriceps muscle in partially flexed knee with the foot on the ground. Mechanism of injury is most frequently caused by fall and direct trauma (Ellanti et al., 2016). In elderly, the degenerative changes associated with ageing and calcific tendinopathy have been shown to be a cause of quadriceps tendon ruptures (Maffulli et al., 2012; Oliva et al.,
2012). Diabetes, chronic renal failures, gout, pseudogout, obesity, hyperparathyroidsm, systemic lupus erythematosus, steroid use, and antibiotic quinolone use have been shown to be related with quadriceps tendon rupture (Shah, 2002; Levy et al., 1971; Preston, 1972; Tedd et al., 2000; Khaliq and Zhanel, 2005; Zribi et al., 2018). This injury are uncommon and there are only103 literatures reporting simulatenous bilateral quadriceps tendon rupture in PubMed search engine. In this literature we reported a case of bilateral quadriceps tendon rupture in a healthy elderly woman.

\section{CASE REPORT}

The patient is a 65-year-old female lives in Central Java. The patient is a housewife. 
The patient came to Hospital with chief complain pain in both knees since 4months before entering the hospital. Initially the patient was slipped and fell with her both knees hit the ground four months ago.She complained the pain was getting worse and after that she could not be able to walk. Her ambulation was with wheel chair, there is no history of fever, no weight loss, normal appetite. The patient has no history of other diseases such as diabetes, hypertension, and other metabolic diseases. The patient does not have a history of the same disease on his family.
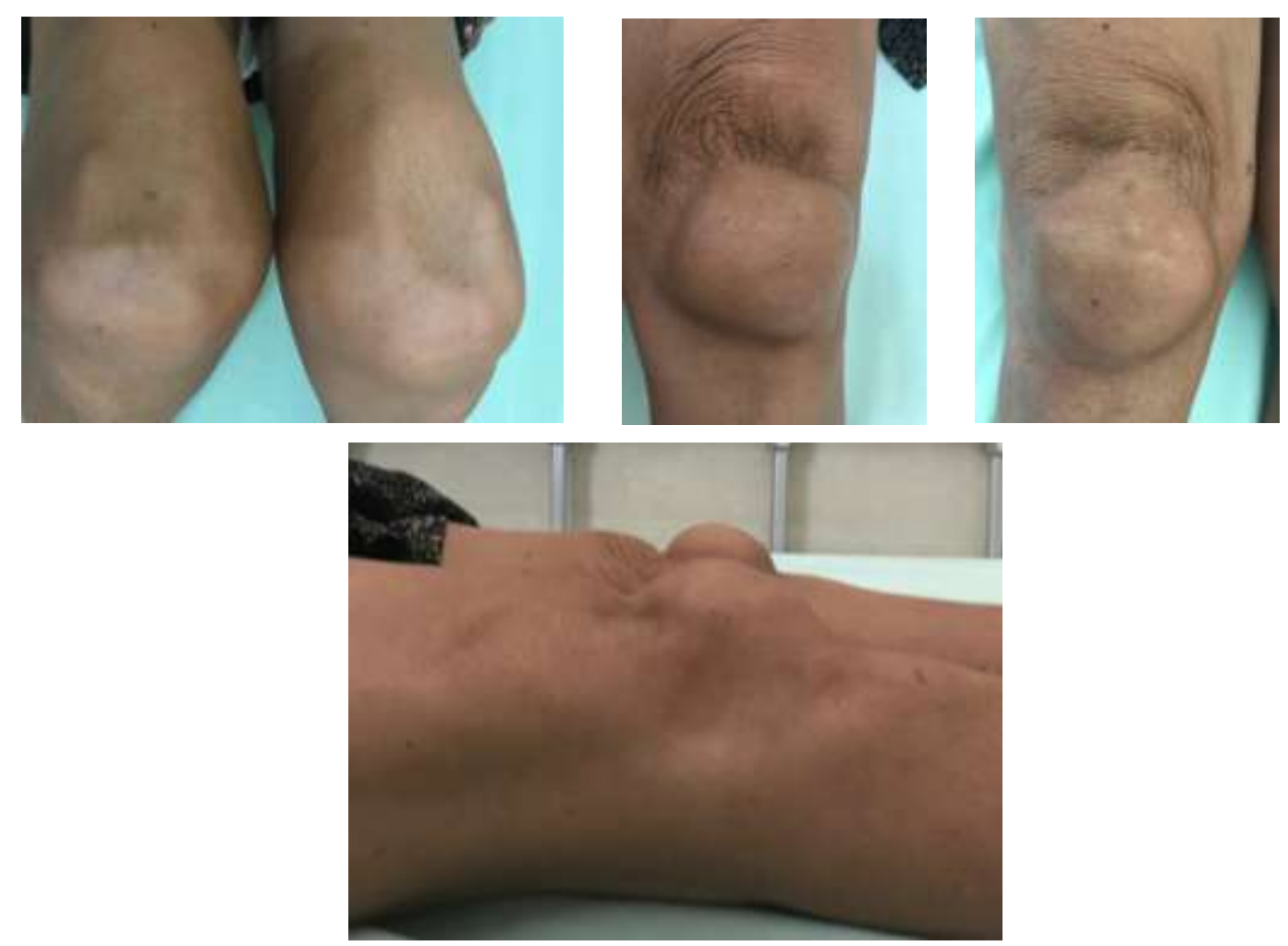

Figure 1. Physical Examination

On physical examination the patient could not be able to walk, in general condition, the patient was not obese andthere is no obvious abnormality. Local physical examination on both knees reveal the loss of normal contour of rectus femoris, tenderness at superior pole patella the ruptur site, palpable defect within $2 \mathrm{~cm}$ of superior pole patella. Patient unable to extend knee against resistance, and unable to perform straight leg raise.

From radiograph examination the AP and lateral knee view shows patella Baja. The patient was carried out a surgical repair with consent. Patient in general anesthesia in supine position, incision was carried out about $7 \mathrm{~cm}$ long midline to knee. The incision is layer by layer up to the quadricep tendon, then the total quadricep tendon rupture was identified. Drill holes in patella, then used non absorbable sutures in tendon in a running locking fashion with ends free to be passed through osseous drill holes until the gap decrease. Retinaculum is repaired too. After that we checked the knee could flex to 90 degrees. The same procedure was done on the contralateral site. The same defect was identified and the same procedurewas done to decrease the gap, repair the defect and the knee could flex to 90 degree after repair. After the surgery 
both knees were immobilized with knee extension splint for 6 weeks.

Patients was treated in ward and were given antibiotics, analgesics, and wound care regularly. The patient underwent immobilization with non weight-bearing and extension of the knee for three weeks.
After six weeks, patient started physiotherapy to improve range of motion with passive and active movement. Patient evaluation revealed no pain and progressive recovery of muscular strength after four months and could be able to walk and returned to normal activity.

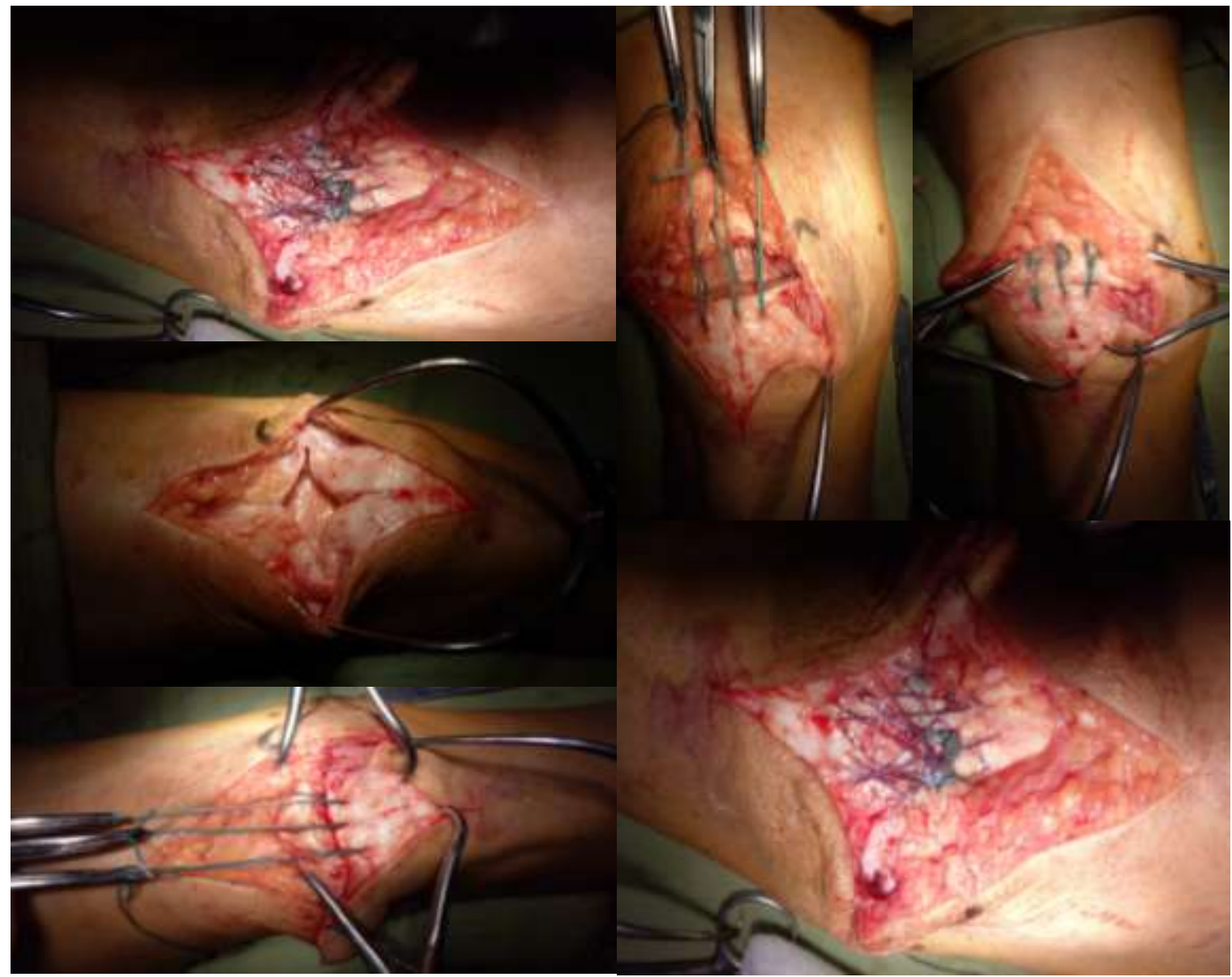

Figure 2. Intra operative

\section{DISCUSSION}

Bilateral rupture of quadriceps tendon is a rare injury. Some literatures have been describe the predisposing factors such diabetes, chronic renal failures, gout, pseudogout, obesity, hyperparathyroidsm, systemic lupus erythematosus, steroid use, and antibiotic quinolone use have been shown to be related with quadrieps tendon rupture (Shah, 2002; Levy et al., 1971; Preston, 1972; Tedd et al., 2000; Khaliq and Zhanel, 2005; Zribi et al., 2018). Patient usually come to hospital with complain of severe knee pain or in neglected case they complain about functional disability of lower limbs. In our case with simultaneous bilateral rupture, the patient could not be able to walk. The mechanism injury usually direct or indirect trauma with knee flexes and foot is planted on the ground, or explosive contraction of quadriceps tendon. In younger patients, sometimes major trauma by direct shock or blunt object are more common in athletes. However, in 
elderly patient, the reduce of tendon elasticity will cause the tendon rupture. In previous case reports, weakening tendon were caused by bone resorption at tendon insertion sites due to hyperparathyroidism (Preston, 1972; Zribi et al., 2018). Collagen was replaced by elastin during chronic metabolic acidosis in chronic renal failure and weakened the tendon and caused rupture (Zribi et al., 2018). In other study, many cases in athletes consuming anabolic agents such as steroid are reported related to increased risk of tendon rupture (Lewis et al., 2005). However in our study, the patient was in healthy condition, no history of trauma, history of steroid injection or fluoroquinolone use, no history of kidney failure, and no history of hyperparathyroidism.

The diagnosis of quadriceps tendon rupture had been based primarily on history taking and clinical examination (Ziribi et al., 2018). Clinical examination is crucial and allows for rapid diagnosis. In clinical examination, patient could not be able to perform ful active extension of knee (Ramsey and Muller, 1970). When quadricep tendon is torn but the retinaculum intact patient are able to achieve active assisted extension, but not full. In palpation there was a gap about two centimeters above patella in myotendineois junction. However, swelling and hematome will make the gap disseapear in acute condition (Ilan et al., 2003). The same as in our case, the gap was felt because the onset was about one month ago, so the swelling and hematom were already subsided. The gap was about two centimeters above superior pole patella.

Other diagnostic modality are ultrasonography, it is minimal invasive, but the results was by examiner. MRI is another sensitive diagnostic tool that can locate the rupture/tear. As such in our case, we did not use MRI as a diagnostic tool prior to surgery. Various surgical technique have been developed such as Krackow procedure, Lightheart procederure. After the operation, walking was permitted, splint was applied for 3-6 weeks to protect the suturing. Early mobilization was perfomed with splint (Ennaciri et al., 2015).A missed diagnosis may lead to delayed repair, which could be problematic due to significant quadriceps retraction. As a result, end-toend tendon suturing becomes difficult. Early diagnosis and treatment are therefore essential for good healing and functional recovery.

Simultaneous ruptures of the quadriceptendon bilateral are rare, which result in disability of both lower limbs. Early diagnosis allows for rapid surgical management and thus optimal functional recovery, careful investigation of such ruptures through clinical examination, plain radiographs, and MRI is therefore necessary for better analysis.

\section{REFFERENCE}

Clayton RA, Court-Brown CM (2008). The epidemiology of musculoskeletal tendinous and ligamentous injuries. Injury. 39(12):1338-44.

Ennaciri B, Montbarbon E, Beaudouin E (2015). Surgical management of acute quadriceps tendon rupture (a case report with literature review). Pan Afr Med J. 22: 243.

Ellanti P, Davarinos N, Morris S, Rice J (2012). Bilateral synchronous rupture of the quadriceps tendon. Ir J Med Sci. 181(3):423-5.

Ellanti P, Moriarty A, Nagle M, McCarthy T (2016). Outcomes after quadriceps tendon repair in patient over 80 years of age. Muscle Ligaments Tendons J. 6(2):224-227. 
Indonesian Journal of Medicine (2019), 4(1): 54-58

https://doi.org/10.26911/theijmed.2019.04.01.09

Ilan DI, Tejwani N, Keschner M, Leibman $M$ (2003). Quadriceps tendon rupture. J Am Acad Orthop Surg. 11(3): 192-200.

Khaliq Y, Zhanel GG (2005). Musculoskeletal injury associated with fluoroquinolone antibiotics. Clin Plast Surg. 32(4):495-502.

Levy M, Seelenfreund M, Maor P, Fried A, Lurie M (1971). Bilateral spontaneous and simultaneous rupture of the quadriceps tendons in gout. $\mathrm{J}$ Bone Joint Surg Br. 53(3): 510-513.

Lewis AC, Purushotham B, Power DM (2005). Bilateral simultaneous quadriceps tendon rupture in a body builder. Orthopedics. 28(7):701-2.

Maffulli N, Del Buono A, Spiezia F, Longo UG, Denaro V (2012). Light microscopic histology of quadriceps tendon ruptures. Int Orthop. 36(11): 23672371.

Oliva F, GiaiVia A, Maffulli N (2012). Physiopathology of intratendinous calcific deposition. BMC Med. 10:95.

Preston ET (1972). Avulsion of both quadriceps tendons in hyperparathyroidism. The Journal of the American Medical Association. 221(4): 406407. doi: 10.1001/jama.221.4.406.
Ramsey RH, Muller GE (1970). Quadriceps tendon rupture: a diagnostic trap.Clin Orthop Relat Res. 70: 161-4.

Senevirathna S, Radha S, Rajeev A (2011). Bilateral simultaneous rupture of the quadriceps tendon in a patient with psoriasis : a case report and review of the literature. J Med Case Reports. 5; 331.

Shah MK (2002). Simultaneous bilateral rupture of quadriceps tendons: Analysis of risk factors and associations. South Med J. 95(8):860-866.

Tedd RJ, Norton MR, Thomas WG (2000). Bilateral simultaneous atraumatic quadriceps tendon ruptures associated with 'pseudogout' Injury. 31(6): 467-469. doi: 10.1016/soo20-1383(oo)00011-5.

Zribi W, Zribi M, Guidara AR, Ben Jemaa M, Abid A, Krid N, Naceur A, Keskes $H$ (2018). Spontaneous and simultaneous complete bilateral rupture of the quadriceps tendon in a patient receiving hemodialysis: A case report and literature review. World J Orthop. 9(9): 180-184. doi: 10.5312/wjo.v9.i9.180. 